UDC 535.421, 535.44, 535015

PACS 42.25.Fx, 42.62 Fi

DOI: $10.22363 / 2658-4670-2019-27-2-143-153$

\title{
Characteristics of optical filters built on the basis of periodic relief reflective structures
}

\author{
Vladislav A. Komotskii ${ }^{1}$, \\ Jose Anibal Pauyac Huaman ${ }^{2}$, Valeriya D. Evstigneeva ${ }^{1}$ \\ 1 Institute of Physical Research and Technologies \\ Peoples' Friendship University of Russia (RUDN university) \\ 6 Miklukho-Maklaya St., Moscow, 117198, Russian Federation \\ 2 Universidad Nacional de Ingenieria \\ Av. Tupac Amaru 210 Rimac, Lima Peru
}

(received: March 15, 2019; accepted: November 14, 2019)

A scheme of a new type optical filter, built using a relief reflective periodic diffraction structure, which has a specific rectangular profile, is proposed. The input radiation beam is directed to the relief structure at a certain angle of incidence. The zero diffraction order beam is our output beam, which is separated from the other diffraction order beams with the help of a diaphragm. The incidence-reflection plane is parallel to the relief lines of the diffraction structure. The dependence of the output beam power on the angle of incidence and on the wavelength of the radiation is investigated. It is shown that the power transfer coefficient from the input to the output of the scheme substantially depends on the wavelength of the optical beam. The scheme can be used as an optical signal filter. The spectral characteristic of this type of filter has an oscillating character. The zero (minimum) values of the power transfer coefficient of radiation from the input to the output of the filter alternate with maximum values close to unity. The spectral characteristic of the filter is easy to change by changing the angle of incidence of the input beam to the relief reflecting structure. Filters of this type can be built for the ultraviolet, visible, and infrared range. Calculations of the dependence of the filter parameters on the relief depth and on the angle of incidence of the input optical beam to the relief structure are presented.

Key words and phrases: filtering of the optical spectrum, diffraction structure, rearrangement of the spectral characteristics of the filter

\section{Introduction}

The need of filtering optical radiation appears in the conduction of physical experiments and in solving technical problems, in which it is necessary to separate a useful signal with specific wavelength from the background radiation 
or eliminate a certain part of the spectrum that interferes with measurements [1]. Filters based on colored glasses [2], filters built using dye solutions, as well as filters built using multilayer dielectric coatings [3] are widely used in practice. However, the above types of filters do not have the capability to rearrange their spectral characteristics. To rearrange the spectral characteristics is possible in acousto-optic filters [4], but it is difficult to manufacture filters of this type, and they are expensive devices.

In this paper, we consider the spectral characteristics of optical filters of a new type [5], which are constructed using relief reflective structures (RRS) with a rectangular profile, with "square wave" form, whose protrusion length is equal to depression length. The RRS profile is shown in Figure 1. The depth of the relief structure $H_{g}$, usually lies in the range from half the wavelength to several wavelengths of optical radiation in a given range. Relief structures of this type can be fabricated on a glass substrate using the method of photolithography and etching, and then covering the structure with metal thin film having high reflection coefficients [6]. Also, polished metal plates with high reflectance in a given spectral region can be used as substrates. For example, silver or aluminum [7]. The range of wavelengths in which the implementation of filters of this type is possible is quite wide: it includes part of the ultraviolet range, the visible range and part of the infrared range.

A characteristic feature of filters of this type is the possibility to change its spectral characteristics by changing the angle of incidence of the input optical beam to RRS. The dependence of the power transfer coefficient on the radiation wavelength, in filters of this type, has smooth shape with a certain number of minima and maxima. At the minima, the transfer coefficient is equal or close to zero, and at the maxima, the transfer coefficient is equal to the reflection coefficient from the surface of the metal thin film or a metal plate on which the RRS is located. It should be mentioned that relief structures with a rectangular shape have been used as optical filters before [8]. But a scheme was considered, in which the optical beam passed through a transparent substrate, with a transparent relief structure on its surface. For such structures, the dependence of the transmission coefficient on the wavelength is similar to the dependences that we describe in this paper. However, it is quite difficult to change the spectral characteristics of the filter changing the angle of incidence, because when we change the angle of incidence, it is necessary to rearrange the scheme. Thus, this paper focuses on the possibility of changing the spectral characteristics of filters when the angle of incidence of the input optical beam is changed.

\section{Filter Scheme, Basic Relations for Calculating Characteristics}

The filter scheme is shown in Figure 1.

The radiation source can be a laser (1), or a light source with a collimator forming a parallel optical beam. The radiation beam from the source is directed to the reflecting relief structure (2) at an angle $\theta$, so that the incidence-reflection plane is parallel to the grooves forming the RRS. After reflection from the RRS, the radiation beam breaks down into diffraction orders and is directed to the diaphragm (3). Only zero diffraction order passes 
the diaphragm, all higher diffraction orders are cut off. Zero order beam is the output beam in this filter. The power transfer coefficient of the filter is defined as

$$
k_{p}=\frac{P_{0}}{P_{\text {in }}},
$$

where $P_{\text {in }}$ is the radiation power at the input of the optical scheme, and $P_{0}$ is the zero diffraction order radiation power at the output of the optical scheme.

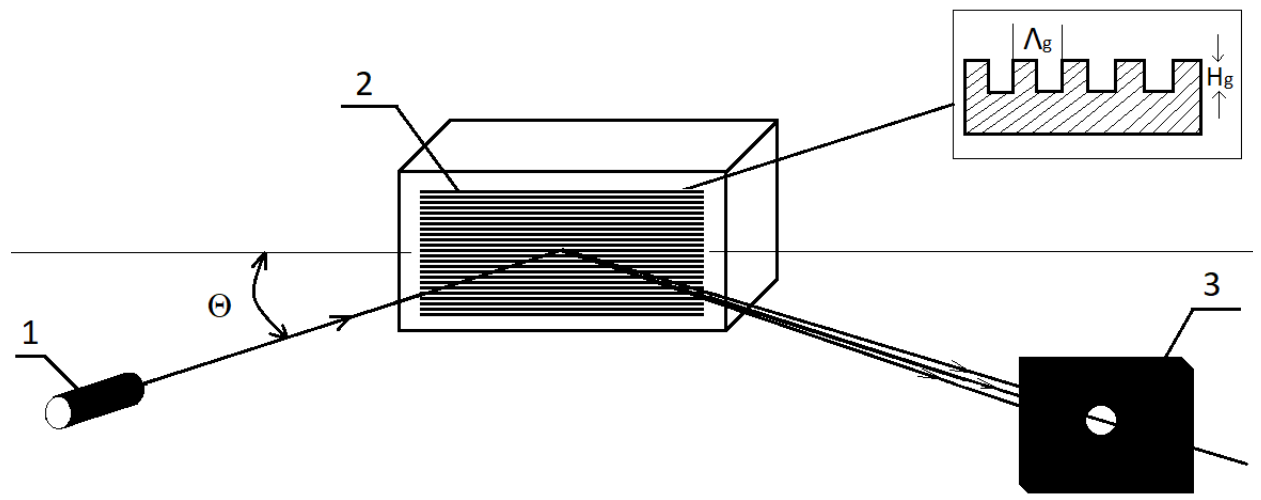

Figure 1. Filter scheme. 1 - radiation source, 2 - relief reflective structure (RRS), 3 - diaphragm

The spatial spectrum of an optical wave after reflection from the RRS consists of a set of diffraction orders. In this scheme we separate only the zero diffraction order. A detailed analysis of the spatial spectrum during the diffraction of an optical wave by phase gratings, which create a rectangular shape modulation of the wave-front phase, is given in [9]. We choose, in this work, periodic reflecting gratings with rectangular profile of the "square wave" form, in which the width of the protrusion is equal to the width of the depression. After wave diffraction on such a structure, there are no even orders in the spatial spectrum. The formulas for calculating the powers of the diffraction orders are given in the appendix. From the analysis of these formulas it follows that when the RRS depth varies in the range from zero to several wavelengths, the power of the zero diffraction order changes from zero to the maximum value.

For different wavelengths, and for different angles of incidence, the power transfer coefficient from input to output of the scheme is different. Dependence of the transfer coefficient on the radiation wavelength $\lambda$, angle of incidence $\theta$, and RRS depth $H_{g}$ for zero diffraction order, can be expressed by the formula:

$$
k_{p}=\frac{P_{0}}{P_{\text {in }}}=R\left(0.5+0.5 \cos \left(\frac{4 \pi}{\lambda} H_{g} \cos \theta\right)\right) .
$$

Here $R$ is the reflection coefficient of the RRS surface.

As follows from formula (2) at a given relief depth $H_{g}$, and at a given angle of incidence $\theta$, the dependence of the transfer coefficient on the wavelength $\lambda$, is determined by a function of the form: $\cos \left(\lambda^{-1}\right)$. The transfer coefficient can take the minimum values equal to zero and the maximum values equal to 
reflection coefficient $R$. If the relief structure is made on a glass substrate, in order to obtain high values of the transfer coefficient at the maxima, we cover the structure with a metal thin film with a high reflection coefficient $R$.

From formula (2) it can be derived that the zero (minimum) values of the transfer coefficient correspond to the following wavelengths:

$$
\lambda_{\min }=\frac{4 H_{g}}{(2 n+1)} \cos \theta, \quad(n=0,1,2, \ldots) .
$$

The maximum values of the transfer coefficient correspond to the following wavelengths:

$$
\lambda_{\max }=\frac{2 H_{g}}{k} \cos \theta, \quad(k=1,2,3, \ldots) .
$$

As can be seen from formulas (3) and (4), the spectral characteristics of the filter can be changed by changing the angle of incidence of the optical beam to the RRS. But, since changing the angle of incidence of the input beam changes the angle of the reflected optical beam, then in order to separate the zero diffraction order in the scheme shown in Figure 1, it will be necessary to change the position of the diaphragm (3).

As shown in Figure 2, another filter scheme is possible, in which the direction of the output beam does not change when the angle of incidence of the input beam changes. The input beam is directed from radiation source (1) to the RRS (2), which is located on one of the faces of the corner reflector. A mirror is located on the other face of the corner reflector. The angle between the RRS plane and the mirror plane is $90^{\circ}$. The beam reflected from the mirror is directed to the diaphragm (4), which permits the passing of only the zero diffraction order and does not permit the passing of higher orders. When the corner reflector is rotated, the angle of incidence of the input beam changes related to RRS. This changes the spectral characteristic of the filter. But the direction of the output optical beam relative to the base coordinate system and relative to the input optical beam does not change.

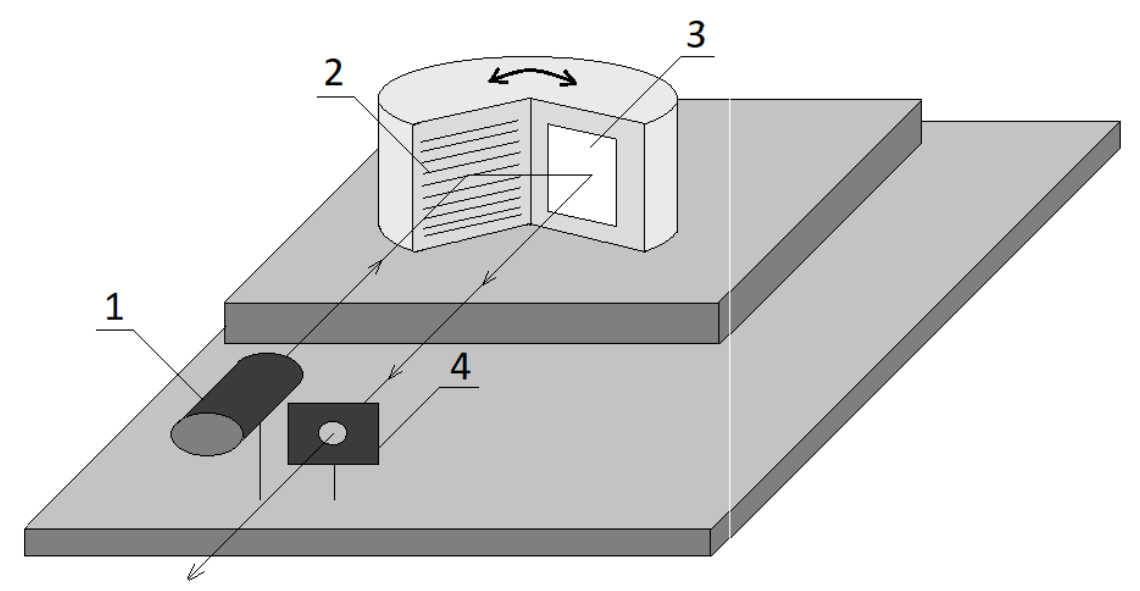

Figure 2. Scheme of the filter using a corner reflector. 1 - radiation source, 2 - RRS, 3 - mirror, 4 - diaphragm 
However, in practice, the use of a corner reflector limits the range of angles of incidence of the input beam to RRS. If the angle of incidence of the input beam is $45^{\circ}$, then the point of incidence of the input beam at RRS and the point of reflection of the beam from the mirror (3) are at the same distance from the plane of symmetry of the corner reflector. Let us assume that the axis of rotation of the corner reflector passes through the point of incidence of the input beam on the RRS. Then, when the corner reflector is rotated, the position of the point of incidence on the RRS remains unchanged, and the reflection point at the mirror moves, and at certain angles of rotation it may go out from the mirror limits, and as a consequence the scheme would stop working. Thus, the scheme shown in Figure 2 has smaller range of possibilities for changing the spectral characteristics of the filter compared with the first scheme (Figure 1).

\section{Estimated characteristics of filters based on RRS}

Figure 3 shows the dependence of the transmission coefficient on the wavelength, estimated for RRS with a relief depth of $H_{g}=0.4 \mu \mathrm{m}$ at three values of the angle of incidence $\left(20^{\circ}, 40^{\circ}, 60^{\circ}\right)$.
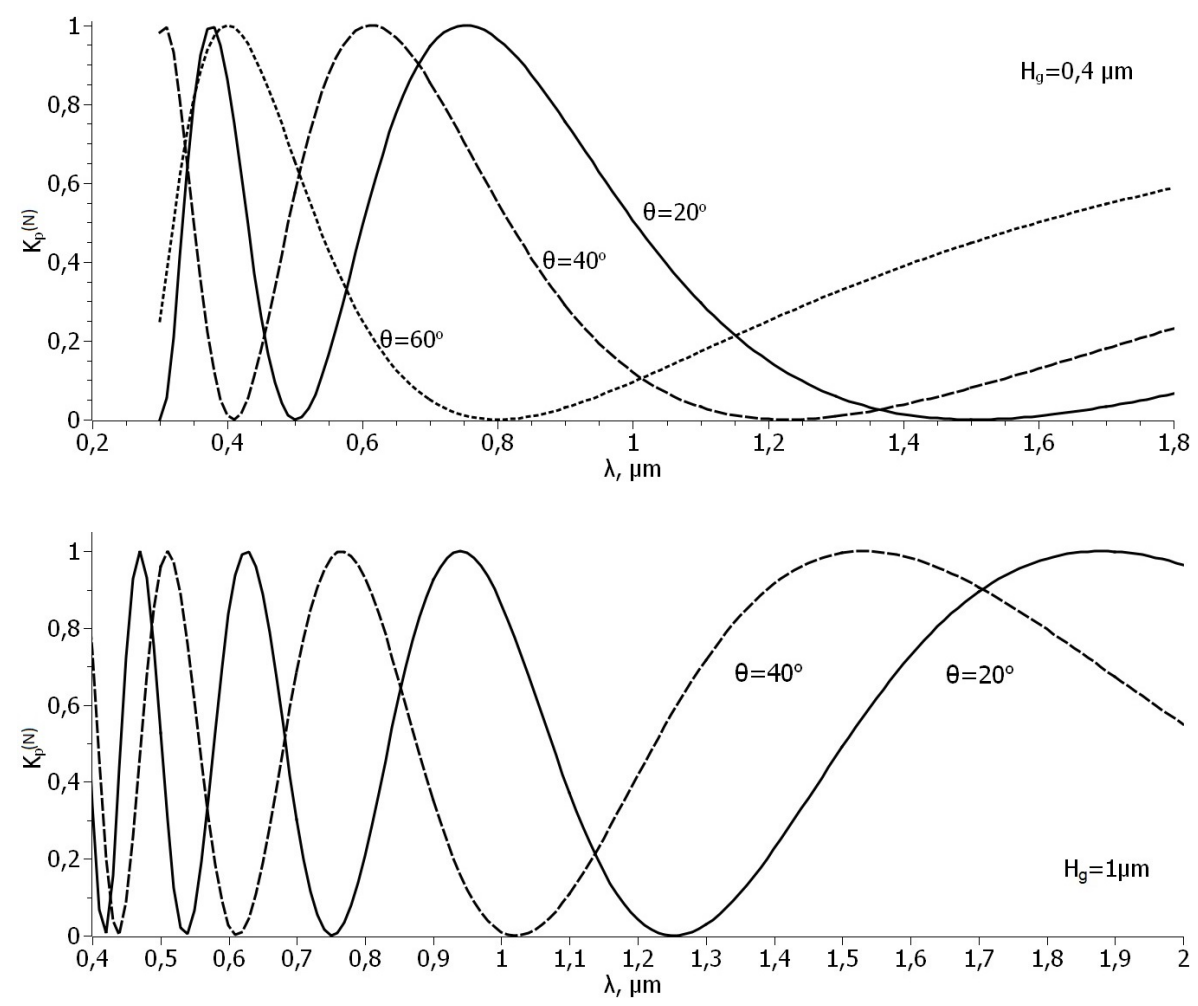

Figure 3. Dependence of the normalized transmission coefficients of the filters on the incident beam wavelength. The upper graph (a) corresponds to the depth $H_{g}=0.4 \mu \mathrm{m}$ at different angles of incidence $\theta=20^{\circ}, 40^{\circ}$ and $60^{\circ}$. The bottom graph (b) corresponds to the depth $H_{g}=1 \mu \mathrm{m}$, at angles of incidence $\theta=20^{\circ}$ and $40^{\circ}$ 
The curves are normalized, i.e. the vertical axis represents the value $k_{p}^{(\mathrm{N})}=k_{p} / R$. In other words, the curves correspond to the transfer coefficient, provided that the reflection coefficient of the RRS surface would be equal to unity. As can be seen from the graphs, even with small changes in the angle of incidence, it is possible to obtain significant changes in the spectral characteristics of the transfer coefficient. For example, you can set the task: to completely suppress a certain wavelength. Substituting the value of the wavelength in the formula (3), we calculate the angle at which the transfer coefficient will be zero. Thus we find the solution to this problem. Practically fine tuning of the angle of incidence can be carried out according to the measurement result of the output signal.

Analyzing the dependencies presented in the graphs, you can notice the following features:

- the number of maxima and minima in a certain range of wavelengths increases with increasing of the RRS depth;

- the distance between adjacent maxima and minima decreases with decreasing of wavelength;

- as the angle of incidence increases, the minima and maxima of the curves move toward shorter waves.

At RRS depths more than 1 micron, the curves have several minima and maxima in the visible region of the spectrum.

In order to get a more general idea of the positions of the maxima and minima in a wide wavelength region and for different angles of incidence of the input beam, we constructed the diagrams of the positions of the maxima and minima on the plane of the coordinates $\lambda-\theta$, which are shown in Figure $4(\mathrm{a}, \mathrm{b})$.

Using the diagrams given in Figure 4(a,b), it is easy to estimate how the spectral characteristic of the filter changes as the angle of incidence of the input optical beam changes. If we draw a horizontal line at a given angle of incidence, then the projections onto the horizontal axis of the points of its intersection with the lines of maxima and minima give the corresponding coordinates of minima and maxima in the dependence of the transfer coefficient on the wavelength $\left(k_{p}^{(\mathrm{N})}(\lambda)\right)$.

The longest wavelength corresponding to a minimum of the dependence $\left(k_{p}^{(\mathrm{N})}(\lambda)\right)$, at a certain angle of incidence $\theta$, can be determined by setting $n=0$ in expression (3). This wavelength is: $\lambda_{\min }(n=0)=4 H_{g} \cos \theta$. In the region of wavelengths exceeding this $\lambda_{\min }$, the curve $\left(k_{p}^{(\mathrm{N})}(\lambda)\right)$ have a monotonic increasing character and asymptotically tend to the level $\left(k_{p}^{(\mathrm{N})}(\lambda)\right)=1$. At a wavelength equal to $\lambda_{\max }(k=1)=2 H_{g} \cos \theta$ is located the maximum of the transfer coefficient closest to the longest wavelength corresponding to minimum $\left(\lambda_{\min }(n=0)\right)$. In the region of wavelengths exceeding $\lambda_{\max }(k=$ $1)=2 H_{g} \cos \theta$, we observe a smooth curve with one minimum.

In the region where the wavelength satisfies the condition $\lambda \ll 2 H_{g} \cos \theta$, there are frequent oscillations of the transfer coefficient as the radiation wavelength changes. Using this region you can build a filter that suppresses one radiation wavelength and passes another radiation wavelength, and these wavelengths are close to each other. As an example, we give the characteristic of the filter, using the RRS with a depth of 3 microns. Figure 5 shows the 
dependence of the transmission coefficient on the wavelength of such a filter at two different angles of incidence.
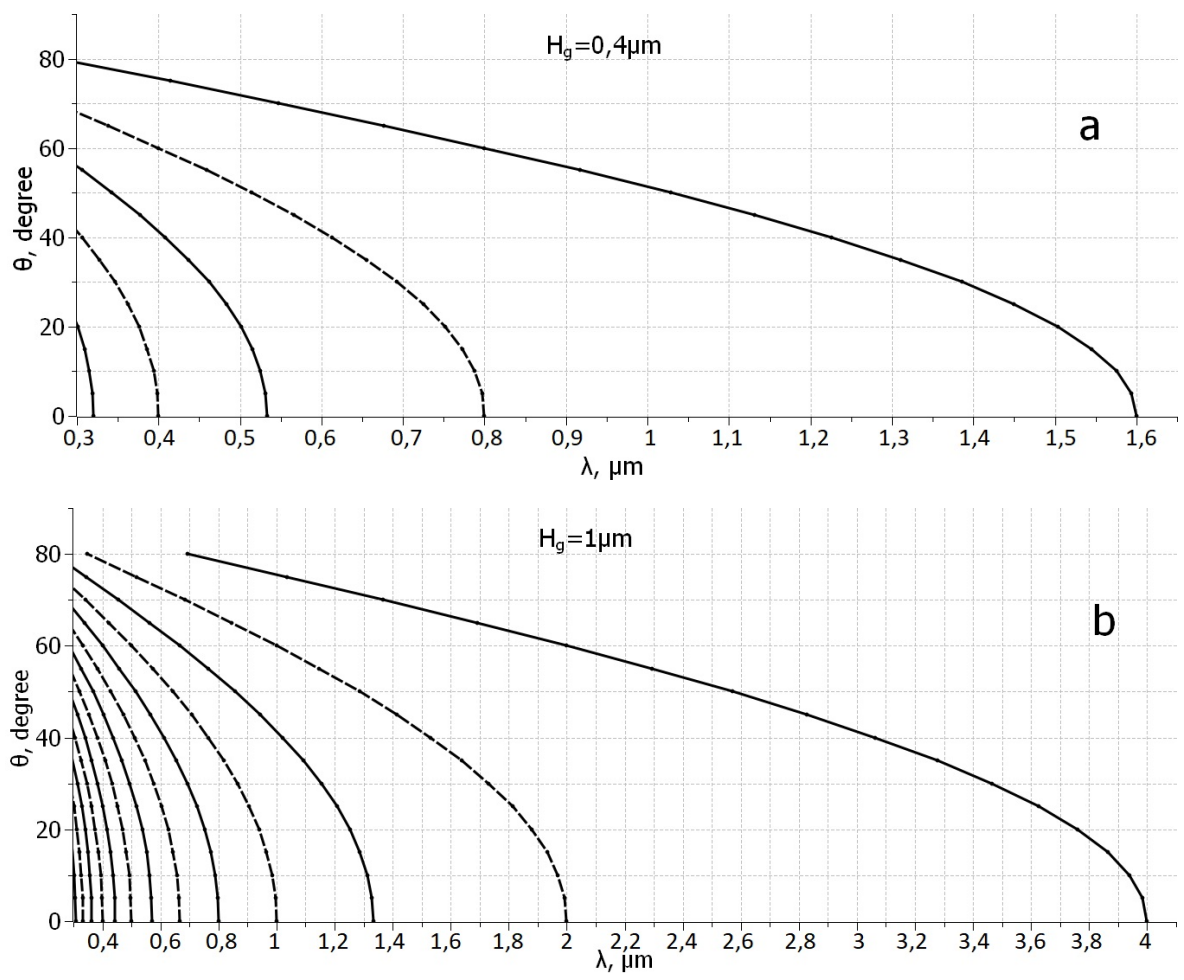

Figure 4. (a, b). Diagram of the positions of the maxima and minima of the output beam power in the zero diffraction order, on a scale of wavelengths, depending on the angle of incidence of the optical beam, for a relief depth equal to: $0.4 \mu \mathrm{m}$ (a) and $1 \mu \mathrm{m}$ (b). The solid lines indicate the positions of the minima, and the dotted lines indicate the positions of the maxima

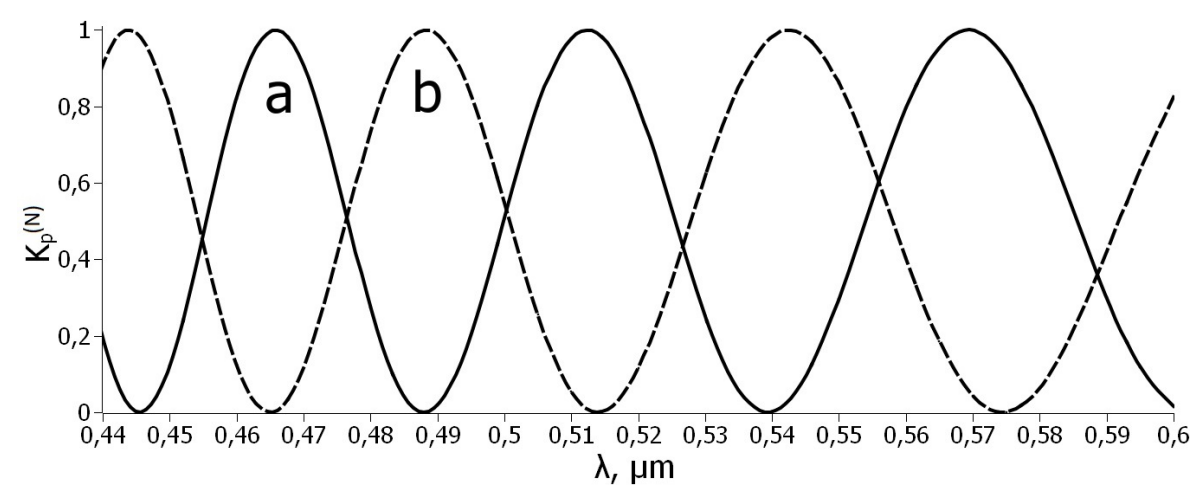

Figure 5. (a, b). Dependence of the transmission coefficient of the filter constructed using RRS on the radiation wavelength. Graph (a): $H_{g}=3 \mu m, \theta=31.35^{\circ}$. Graph (b):

$$
H_{g}=3 \mu m, \theta=35.53^{\circ}
$$


Let us assume that an argon laser beam is directed to our filter. It is known that the argon laser has two strong emission spectral lines: at $0.488 \mu \mathrm{m}$ and at $0.514 \mu \mathrm{m}$ [10]. When the angle of incidence is $\theta=31.35^{\circ}$, radiation with $0.488 \mu \mathrm{m}$ wavelength will not pass to the output of the filter, and for radiation with $0.514 \mu \mathrm{m}$ wavelength the normalize transfer coefficient will be close to 1 .

If you change the angle of incidence and set it equal $\theta=35.53^{\circ}$, then the situation will change to the opposite. The transmission coefficient at a wavelength of $0.514 \mu \mathrm{m}$ will be equal to zero, and the transmission coefficient at a wavelength of $0.488 \mu \mathrm{m}$ will be close to the maximum.

It should be noted that the part of the radiation power that did not pass to the filter output in the zero diffraction order is distributed between the first and higher diffraction orders and is absorbed by the diaphragm.

Consider another example of constructing a filter for wavelength ranges of part of the visible and ultra violet light. As a light source we will consider a mercury lamp. The spectrum of a mercury lamp contains a series of lines with wavelengths in the range from $280 \mathrm{~nm}$ to $630 \mathrm{~nm}$ [11]. These wavelengths are indicated by thin vertical lines in Figure 6 , which also shows the theoretical dependences of the transmission coefficient of two kinds of filters on the wavelength. The filter of the first type, whose spectral characteristic is drawn with a dotted line, is made as a RRS on the surface of a polished aluminum plate, which reflectance is about $90 \%$. This filter can pass two groups of lines: one in the region $280-320 \mathrm{~nm}$ and the other in the region $540-620 \mathrm{~nm}$. At the same time, the intensity of the lines in the region of $360-440 \mathrm{~nm}$ is significantly reduced. The filter of the second type, the spectral characteristic of which is drawn by a solid line, is made on the surface of a polished silver plate. The reflection coefficient of silver in the region of wavelengths less than $350 \mathrm{~nm}$ has a sharp decline. With this in mind, the transmission coefficient of this type of filter has a much smaller value in the region of $280-320 \mathrm{~nm}$ compared with the transmission coefficient of the filter of the first type. In both types of filters, the relief depth was assumed to be $405 \mathrm{~nm}$ and the angle of incidence was $41.4^{\circ}$.

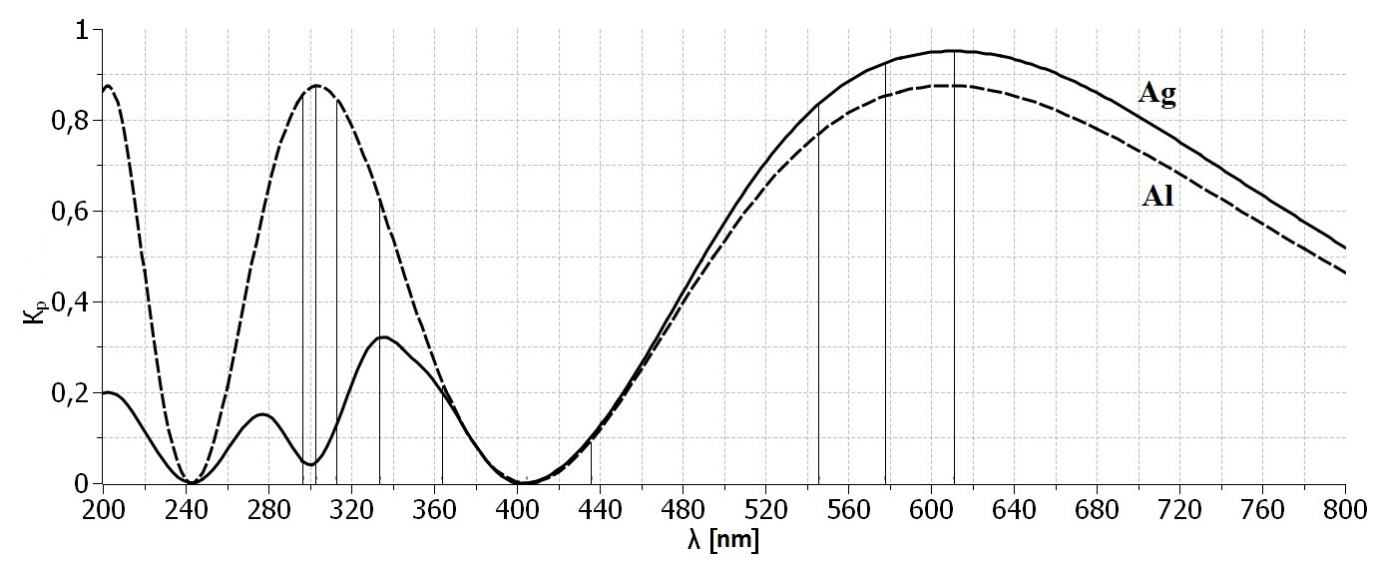

Figure 6. Dependence of the transmission coefficients of the two types of filters built using RRS on the wavelength. The RRS depth is $405 \mathrm{~nm}$. The angle of incidence of the radiation is $41.4^{\circ}$. The solid line corresponds to the RRS on a silver plate, and the dotted line corresponds to the RRS on an aluminum plate 
Parameters such as the RRS period and the diameter of the optical beam are not directly included in the above calculation formulas, therefore, we will discuss here suggestions for choosing these parameters.

The period of the diffraction structure must be significantly longer than the maximum wavelength of the wavelength range in which the filter is supposed to be used. The transverse size of the optical beam must be several times greater than the radiation wavelength, in order for the diffraction beams directions of zero and first orders to be successfully separated in space.

For separation of diffraction beams at the plane of the diaphragm, it is necessary that the distance from the RRS to the diaphragm satisfy the condition:

$$
L=p D \frac{\Lambda}{\lambda}
$$

$L$ is the distance from the RRS to the diaphragm, $D$ is the diameter of the optical beam, $\Lambda$ is the period of the diffraction structure, the number $p$ is the safety factor (no less than 2), which is necessary to prevent penetration of the radiation beams of the first orders of diffraction into the aperture of the diaphragm.

\section{Conclusion}

Filters based on RRS have a number of positive properties. Their spectral characteristics can be easily changed by changing the angle of incidence of the optical beam to the RRS. The design of the filter is quite simple. The relief structure is fabricated using well-developed photolithography and etching technologies. For the manufacture of filters designed to work in the infrared wavelength range, there is no need to use special materials. Regular polished metal plates are quite suitable: aluminum, copper, silver. The transfer coefficient at the maximum is equal to the reflection coefficient of the RRS surface.

Also, we should mention a number of disadvantages of filters of this type. The spectral characteristics of filters of this type are smooth. Their shape is far from rectangular. In this respect they cannot be compared with widely known filters based on multilayer dielectric structures. As can be seen from the principle of operation, filters based on the RRS will work well when they are irradiated by directed laser beams with a small divergence. The question of the interaction of diverging beams with a filter of this type was not considered in this paper.

\section{Appendix}

As a result of the reflection of a coherent optical beam from a relief structure, which has a rectangular profile with "square wave" form, a spatial phase modulation of the wave front takes place. The phase modulation function form is rectangular, and the amplitude of this function is equal to:

$$
\Phi_{M}=\frac{2 \pi}{\lambda} H_{g} \cos \theta
$$


The function of the wave front phase modulation can be expressed as:

$$
f(x)=e^{i \Phi(x),} \text { where } \Phi(x)=\left\{\begin{array}{rll}
\Phi_{M}, & \text { if } & \frac{\Lambda}{2}+k \Lambda>x>0+k \Lambda, \\
-\Phi_{M}, & \text { if } & 0+k \Lambda>x>-\frac{\Lambda}{2}+k \Lambda, \\
k=0,1,2,3, \ldots
\end{array}\right.
$$

We find the spatial spectrum of the coherent optical wave after reflection from the relief structure applying the Fourier transform to this expression. The spatial spectrum consists of a zero order and a set of diffraction orders at spatial frequencies that are multiple of the spatial frequency $\xi=\Lambda^{-1}$.

The expressions for the Fourier coefficients are:

$$
C_{0}=\cos \Phi_{M}, \quad C_{1}=\frac{2}{\pi} \sin \Phi_{M}, \quad C_{m}=\frac{1}{m \pi}\left(\sin \Phi_{M}-\sin \left(\Phi_{M}+\pi m\right)\right) .
$$

The ratio of the radiation power in one diffraction order to the input radiation power $P_{\text {in }}$ is equal to the square of the corresponding Fourier coefficient (assuming that there are no losses).

In particular, the ratio of the power in zero diffraction order to the power at the input of the diffraction structure (provided that the reflection coefficient is $100 \%$ ) is:

$$
\frac{P_{0}}{P_{\text {in }}}=\cos ^{2} \Phi_{M}=0.5+0.5 \cos 2 \Phi_{M}=\left(0.5+0.5 \cos \left(\frac{4 \pi}{\lambda} H_{g} \cos \theta\right)\right) .
$$

\section{Acknowledgments}

The publication has been prepared with the support of the "RUDN University Program 5-100".

\section{References}

1. V. V. Lebedeva, Experimental optics [Eksperimental'naya optika], 3rd Edition, Publishing House of Moscow University, Moscow, 1994, in Russian.

2. V. I. Puchkov, E. A. Iosep, G. T. Petrovsky, L. S. Iutinskaya, A. P. Ivanova, A. V. Smirnova, S. P. Lunkin, Coloured optical glass. Specifications [Steklo opticheskoye tsvetnoye], State Standard of the USSR, GOST 9411-91, http://docs.cntd.ru/document/gost-9411-91, 1991, in Russian.

3. A. M. Prokhorov (Ed.), Handbook of Lasers [Spravochnik po lazeram], Vol. 2, Soviet Radio, Moscow, 1978, in Russian.

4. I. P. Golyamin (Ed.), Ultrasound (small encyclopedia) [Ul'trazvuk (Malen'kaya entsiklopediya)], Soviet Encyclopedia, Moscow, 1979, in Russian.

5. V. A. Komotsky, Y. M. Sokolov, N. V. Suetin, A device for filtering optical signal spectra [Ustroystvo dlya fil'tratsii spektrov opticheskogo signala], Patent for utility model 181381, in Russian (2018). 
6. V. P. Lavrintsev (Ed.), Introduction to photolithography [Vvedeniye v fotolitografiyu], Energy, Moscow, 1977, in Russian.

7. G. V. Rosenberg, Optics of thin-layer coatings [Optika tonkosloynykh pokrytiy], State publishing house of physical and mathematical literature, Moscow, 1958, in Russian.

8. Machine construction encyclopedia XXL [Entsiklopediya po mashinostroyeniyu XXL], in Russian.

URL http://mash-xxl.info

9. N. M. Kashchenko, Determination of the depth of the reference diffraction gratings based on the measurement and analysis of the intensities of the diffraction orders [Opredeleniye glubiny opornykh difraktsionnykh reshetok na osnove izmereniya i analiza intensivnostey difraktsionnykh poryadkov], Bulletin of the Peoples' Friendship University of Russia. Series: Physics 7 (1) (1999) 16-27, in Russian.

10. Gas argon laser LEXEL 85/95 (visible) [Gazovyy argonovyy lazer LEXEL $85 / 95$ (vidimyy)], in Russian.

URL http://scientific-technology.ru/laserslink/gaslaser/ lexel85ar

11. Spectrum of a natural sunshine fluorescent light.

URL

https://commons.wikimedia.org/wiki/File:Spectra-

Philips_32T8_natural_sunshine_fluorescent_light.svg

\section{For citation:}

V.A.Komotskii, J.A.Huaman, V.D.Evstigneeva, Characteristics of optical filters built on the basis of periodic relief reflective structures, Discrete and Continuous Models and Applied Computational Science 27 (2) (2019) 143-153. DOI: 10.22363/2658-4670-2019-27-2-143-153.

\section{Information about the authors:}

Vladislav A. Komotskii (Russian Federation) - Professor, Doctor of Technical Sciences, Professor of Institute of Physical Research and Technology of Peoples' Friendship University of Russia (RUDN University) (e-mail: vkomotskii@mail.ru, phone: $+7(977)$ 4870523, ORCID: https://orcid.org/0000-0002-0997-8182, ResearcherID: E-6416-2018, Scopus Author ID: 6603398628)

Jose Anibal Pauyac Huaman (Peru) - Candidate of Physical and Mathematical Sciences, Intern student of Institute of Physical Research and Technology of Peoples' Friendship University of Russia (RUDN university) (e-mail: jpauyac@hotmail.com, phone: +7 (915) 2330726, ORCID: https://orcid.org/0000-0002-4952-1814)

Valeriya D. Evstigneeva (Russian Federation) — Student of Institute of Physical Research and Technology of Peoples' Friendship University of Russia (RUDN University) (e-mail: evstilera@mail.ru, phone: +7(977) 9569633, ORCID: https://orcid.org/0000-0001-7317-5539) 